\title{
Mixed method study focusing on cost effectiveness of utilizing Guideline Directed Medical Therapy using Secondary and Primary Data
}

Lovekirat Singh'1, Carly Daley², Shauna Wagner², Roy Robertson², Michael Mirro², ${ }^{1}$ Indiana University School of Medicine, ${ }^{2}$ Parkview Health, Department of Cardiology

\section{Background and Hypothesis:}

In the United States, heart failure (HF) affects an increasing 5.8 million people, with healthcare costs estimated at $\$ 31$ billion (2012). Guideline Directed Medical Therapy (GDMT) is the primary method to standardize medical care of patients diagnosed with Heart Failure reduced Ejection Fraction (HFrEF). GDMT lowers mortality rates, decreases readmission, and decreases the cost, but the success of GDMT is limited by adherence. We hypothesize increased GDMT prescription through integration of a pharmacist will decrease readmissions and hospital costs.

\section{Methods:}

This mixed-methods study involved a literature review and retrospective chart review. Online databases (PubMed, Embase, and Ovid) were searched using keywords related to GDMT and economic outcomes. The research was refined using empirical research and grey literature focusing on healthcare costs and readmission rates. The chart review is part of a larger study examining the impact of integrating a pharmacist into a cardiology center on GDMT prescription. Inpatient and outpatient charges data was analyzed to determine if integration of a pharmacist led to decreased healthcare costs. This project was supported by Parkview Health and received funding from ACC Accreditation Foundation Board.

\section{Results:}

The study is currently ongoing. 70 articles and abstracts were reviewed, and 10 articles fit the criteria focusing on GDMT, healthcare cost, or readmissions. Articles supported a correlation between decreased readmission and overall healthcare cost with compliance to GDMT. Literature also supports the addition of an intervention leading to decreased readmissions. Preliminary charges data analysis showed a decrease in cost by $46 \%$ in inpatients and $35 \%$ in outpatients after pharmacist intervention.

\section{Conclusion:}

We conclude GDMT is associated with decreased healthcare costs due to decreased readmissions. Although there are multiple confounding variables when evaluating the primary data, data showed a correlation between adding intervention (i.e. pharmacist) and decreasing readmissions and overall healthcare costs. Further studies need to be conducted to show causation. 